\title{
Modifications de l'OPAS
}

\section{INFO TARIFAIRE IMPORTANTE}

\section{Thomas Kessler, Kerstin Rutsch}

Département Tarifs et conventions pour la médecine ambulatoire en Suisse

\section{Modifications de l'OPAS (ordonnance sur les prestations de l'assurance des soins)}

Plusieurs modifications de l'ordonnance sur les prestations de l'assurance des soins (OPAS) et de ses an-

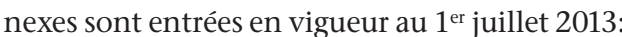

\section{Modifications d'articles de loi}

Vaccinations prophylactiques (art. 12a OPAS): il convient désormais de suivre le Plan de vaccination suisse 2013.

En outre, le dépistage du cancer du côlon (tranche d'âge de 50 à 69 ans) a été intégré comme prestation obligatoire dans l'OPAS (art. 12e let. d).

Selon l'art. 13 let. OPAS, l'assurance prend en charge, en cas de maternité, les examens de contrôle suivants: amniocentèse et prélèvement des villosités choriales. Et ce après un entretien approfondi qui doit être consigné. Pour les femmes âgées de plus de 35 ans, c'est désormais l'âge révolu à la date présumée du terme de la grossesse qui est déterminant.

\section{Modifications de l'annexe 1 de l'OPAS}

Depuis le $1^{\text {er }}$ juillet 2013, les prestations médicales suivantes sont, à certaines conditions, obligatoirement à la charge de l'assurance conformément à l'annexe 1 de l'OPAS:

- traitement de l'incontinence urinaire par injection cystoscopique de toxine botulique de type $\mathrm{A}$ dans la paroi vésicale

- nutrition entérale à domicile sans utilisation de sonde

- intervention par voie percutanée visant à traiter une grave insuffisance mitrale

- implantation transcathéter de valve aortique (TAVI) (en cours d'évaluation)
- traitement de la lipoatrophie du visage par des produits de comblement

- enfin, la tomographie par émission de positrons (TEP) au moyen de F-2-Fluoro-Deoxy-Glucose (FDG) est désormais obligatoirement prise en charge dans le domaine de la neurologie comme mesure préopératoire en cas d'épilepsie focale résistante à la thérapie.

En revanche, les prestations médicales suivantes ne sont plus prises en charge selon l'annexe 1 OPAS:

- traitement des varices par ablation mécanochimique endoveineuse de type Clarivein ${ }^{\circledast}$

- électroneuromodulation des nerfs pelviens à l'aide d'un système implanté par laparoscopie (procédure LION: Laparoscopic Implantation of Neuroprothesis)

- désormais, la réadaptation des patients souffrant de maladies cardiovasculaires ou de diabète n'est plus prise en charge pour les patients souffrant d'une maladie artérielle périphérique (MAP) au stade I selon Fontaine.

Modifications de la liste des analyses (annexe 3 OPAS) et de la liste des moyens et appareils (annexe 2 OPAS)

Une autre modification importante concerne la liste des analyses: le supplément de transition par analyse (4708.00) est prolongé jusqu'au 31 décembre 2013 (PT 1.1 = 1.10 CHF par analyse).

Par ailleurs, l'achat de l'appareil de neurostimulation transcutanée électrique (09.02.01.00.1) figure désormais dans la liste des moyens et appareils (LiMA).

\begin{abstract}
A inscrire dans vos agendas:
Journée des délégués tarifaires du mercredi 23 octobre 2013, HOTELBERN, Berne

Pour la deuxième Journée des délégués tarifaires organisée cette année, nous avons également prévu des exposés et ateliers intéressants. II nous a notamment été possible d'obtenir la venue du Dr Ignazio Cassis, président de curafutura, pour un exposé.

Le paysage tarifaire a connu de nombreux changements - nous vous présenterons les principaux thèmes d'actualité et nous réjouissons d'en discuter avec vous. Les invitations personnelles et le programme détaillé seront envoyés à la fin septembre par courrier électronique.
\end{abstract}

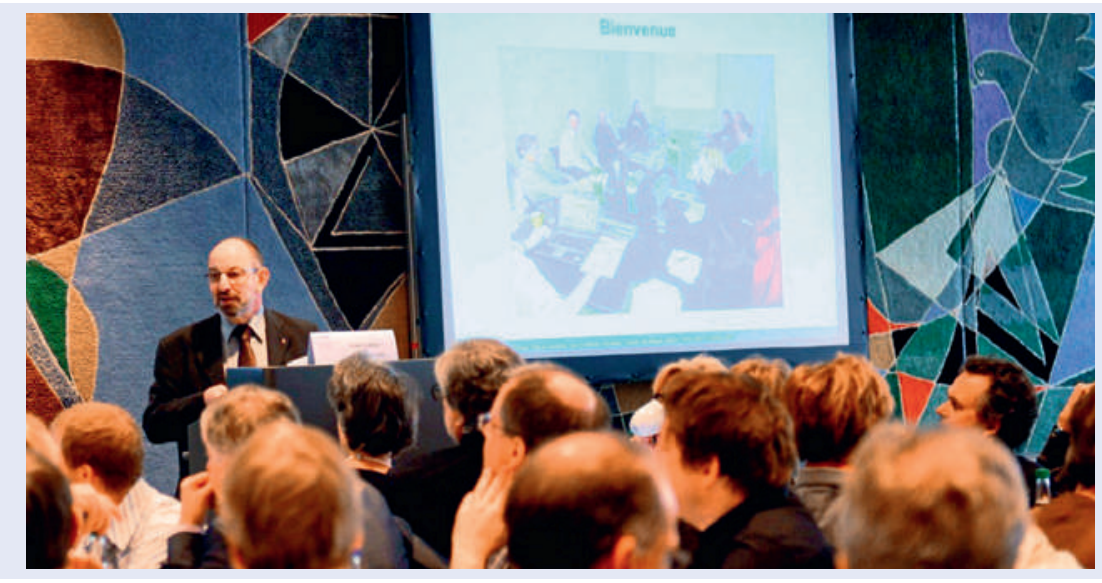

